\title{
Comment se construit l'invisibilité des populations pastorales au Sahel : l'expérience de la crise alimentaire de 2005 au Niger
}

\author{
Véronique Ancey* \\ CIRAD, UMR ART-DEV, TA C-113/15, 73 rue Jean-François Breton, 34398 Montpellier cedex 5, France
}

\begin{abstract}
Résumé - Au Sahel, les systèmes d'information élaborés et perfectionnés depuis les années 1980 n'ont pas suffi aux politiques nationales et aux actions d'urgence internationales pour gérer la spécificité des populations pastorales. Cette inadéquation renvoie aux difficultés historiques des administrations à prendre en compte la gestion commune des ressources naturelles et la mobilité des troupeaux. Elle s'explique en partie seulement par les limites des systèmes d'information, le manque de moyens ou un défaut de coordination au sein des politiques nationales et régionales. À partir de l'expérience de la crise alimentaire de 2005 au Niger et d'une quinzaine d'années de travaux de terrain au Sahel, ce texte montre comment s'est construite « l'invisibilité » des pasteurs : d'un côté, les schémas de pensée sous-jacents aux systèmes d'information n'aident pas les politiques à gérer les spécificités au cœur de la reproduction des systèmes pastoraux. Mais, d'un autre côté, la stratégie d'évitement des populations pastorales qui leur a historiquement permis d'échapper aux contraintes exercées par l'administration les dessert aujourd'hui en compromettant leur capacité à peser sur des débats fondamentaux au sein de leurs pays. Au-delà, le cas singulier du pastoralisme mérite de susciter l'attention de recherches pluridisciplinaires et de politiques prenant en compte une gestion durable des espaces, des ressources et du vivant, dans un monde exposé à de profondes mutations économiques et climatiques.
\end{abstract}

Mots clés : information / évitement / pastoralisme / crise / Sahel / politique agricole

\begin{abstract}
How pastoral populations have become invisible in the Sahel. The experience of the 2005 food crisis in Niger. In the Sahel, the information systems built and improved since the 1980s have not allowed development policies and emergency actions to take into account some specific traits of the pastoral populations. This inadequacy goes back to historical background and partly addresses the limitations of the information systems, the lack of means or the weak coordination within and between national and regional politics. The administration struggles to cope with the common management of natural resources and the mobility of herds. Empirically grounded on a fieldwork following the 2005 food crisis in Niger and on 15 years of field enquiries in the Sahel, this article intends to demonstrate how pastoral populations have become "invisible". On the one hand, the underpinning schemes in the information systems do not help the policies managing the specific traits at the core of the reproduction of the pastoral systems. On the other hand, the pastoral population's avoidance strategy, which has historically allowed them to escape from pressure exerted by the administration, currently hinders their ability to influence fundamental debates within their countries. The singular case of pastoralism should raise awareness of multidisciplinary research and politics concerned with the sustainable management of space, resources and living within a world confronted with deep economic and climate changes.
\end{abstract}

Keywords: information / avoidance / pastoralism / crisis / Sahel / agricultural policies

La tension qui « parcourt toute l'histoire de la statistique » (Desrosières, 2010) entre les points de vue descriptif et prescriptif, et la construction sociale des modèles scientifiques créent des angles morts pour la connaissance. Dans la recherche

\footnotetext{
* Auteur de correspondance : veronique.ancey@cirad.fr
}

agricole, on connaît le biais de genre des enquêtes agroéconomiques sexo-indifférenciées (Verschuur et al., 2015), l'ethnocentrisme de certaines catégories statistiques (Couty, 1984 ; Gastellu et al., 1997) et domestiques (Meillassoux, 1975 ; Ancey et Fréguin-Gresh, 2014), ou encore l'économicisme négligeant les aspects non marchands de la production, des échanges et de la consommation. De plus, une recherche 
spécialisée sur des sujets précis et disciplinaires peine à traiter d'enjeux complexes. En région sahélienne par exemple, de nombreux travaux se référant à la sécurité alimentaire se focalisent en réalité sur des questions plus étroites et ne peuvent embrasser l'ensemble des enjeux : ainsi, l'appréciation spatialisée des niveaux de risques ne correspond pas à la vulnérabilité alimentaire réelle des ménages (Janin et MartinPrevel, 2006). Le ciblage n'améliore pas forcément l'efficacité des actions : Egg et Gabas (1997) ont analysé les difficultés de mise en œuvre des interventions alimentaires ciblées.

Les recherches sur le pastoralisme se sont renouvelées autour des années 1990, en écologie (Behnke et Scoones, 1992 ; Scoones, 1999), en sciences humaines (Khazanov, 1984 ; Marty, 1993 ; Thébaud et al., 1995 ; Swift, 2001) et des bases ont été posées pour écrire l'histoire du développement pastoral (Pouillon, 1990). Cependant, les politiques de développement n'ont guère utilisé ces travaux pour appréhender les caractères spécifiques du pastoralisme, à savoir la gestion intégrée et partagée de l'hétérogénéité et des variations spatio-temporelles des ressources (Krätli et Schareika, 2010), mise en œuvre par les savoir-faire individuels et l'organisation sociale, à travers des faisceaux de droits d'accès à ces ressources et la mobilité des troupeaux. Alors que les capacités adaptatives des systèmes pastoraux et des sociétés qui les animent sont aujourd'hui largement reconnues, les politiques agricoles restent inadaptées à une gestion et un soutien durable de ces systèmes.

À partir d'une connaissance des milieux pastoraux fondée sur une quinzaine d'années de travaux au Sahel, ce texte propose de montrer comment s'est forgée historiquement « l'invisibilité » de cette catégorie d'acteurs du monde agricole et comment elle les dessert en contexte de crise alimentaire. Au-delà de la qualité des informations disponibles, les politiques de développement ont du mal à prendre en compte les caractéristiques des unités écologiques et sociales pastorales, éléments pourtant nécessaires à l'adaptation et à la promotion d'un mode de fonctionnement soutenable. La conception des systèmes d'information est ensuite retracée et leur rôle pendant la crise alimentaire de 2005 au Niger est analysé (Ancey, 2006 ; Egg et al., 2006 ; Olivier de Sardan, 2007, 2008). L'évitement, qui a plutôt servi historiquement les populations pastorales, compromet aujourd'hui leur capacité à peser sur les débats fondamentaux dans leurs pays.

\section{Pourquoi les politiques ont du mal à appréhender l'économie et l'écologie pastorales}

L'observation des interactions entre les pratiques des sociétés pastorales et les idées et politiques du monde du développement renvoie à deux enjeux que les politiques peinent à saisir : la gestion intégrée de ressources naturelles partagées et les mobilités pastorales, autrement dit le rapport entre l'économie et l'écologie pastorales. Cette partie en fournit quelques illustrations et tente de montrer pourquoi les politiques ont du mal à appréhender ces caractéristiques.

\subsection{L'unité écologique et sociale pastorale : une utilisation optimale d'équilibres instables}

Les études scientifiques des pâturages en ont étudié les quantités produites, les valeurs nutritives, la variabilité structurelle des précipitations et des ressources, naturelles ou cultivées (Thornton et al., 2009), la résistance aux sécheresses. Elles sont le plus souvent orientées par une perspective de gestion des ressources et d'aménagement du territoire (Andrieu et al., 2007 ; Steinfeld et al., 2013), ou par les préoccupations autour du changement climatique (Craine et al., 2013). Moins souvent, elles sont conduites sous l'angle de la construction du savoir et de la qualification des ressources par les pasteurs (Figuié et Hubert, 2012). De manière symétrique, les sciences sociales ne peuvent, à elles seules, qualifier les ressources naturelles... Après avoir démontré les interactions entre les pasteurs et le monde qui les entoure, de nouveaux travaux caractérisent aujourd'hui l'écologie pastorale par ses traits internes: des interactions singulières humains-animauxenvironnement. L'hétérogénéité est une ressource et non un obstacle, " caractéristique de l'environnement des terres de parcours, où les ressources clés, nutriments et eau d'abreuvement, se présentent en concentrations imprévisibles et temporaires, et non dans une distribution uniforme et stable. Les aspects cruciaux de la spécialisation pastorale sont : le rôle économique joué par l'interaction humains-animauxenvironnement, en particulier une mobilité stratégique et une alimentation sélective; et le développement de systèmes souples de gestion des ressources, en particulier les institutions de gestion des communs et les droits non exclusifs sur les ressources en eau » (Krätli, 2015). L'optimisation, en pastoralisme, consiste ainsi à utiliser au mieux des équilibres instables, multiples (Sullivan et Rohde, 2002) et suffisants (Hubert, 2012).

En milieux arides et subarides, le droit à la mobilité est donc une ressource stratégique pour les systèmes pastoraux ; elle implique les savoir-faire individuels, l'organisation sociale audelà du pastoralisme, l'environnement institutionnel et les infrastructures. Au Sahel, la distribution des pluies et de la biomasse est aléatoire, spatialement dispersée et variant de $30 \%$ annuellement. La production, l'accès et le partage des informations écologiques et économiques jouent un rôle clé dans la quête des « bons endroits », « modji jofde " ", propices aux belles vaches, lieux non enclos, toujours recherchés. La qualité et la quantité de l'herbe ne suffisent pas à distinguer un endroit attractif pour l'élevage : « Les animaux qui pâturent plus au Sud sont moins jolis qu'ici, pourtant il y a moins d'herbe ici mais l'endroit est de meilleure qualité. » (Rewane, Sénégal). Dans un endroit néfaste, la robe des bêtes change, « comme de la chair de poule » mais si l'endroit est propice, les robes deviennent belles, la production de lait et les mises bas augmentent. " En une semaine sur de bons pâturages, les taureaux commencent à se battre et leur voix change » (Ibid). Les pasteurs utilisent également des ressources invisibles pour les systèmes d'information. Les feuilles tombées, par exemple (samde samde ${ }^{2}$ ), sont précieuses pendant les longues étapes de transhumance sur des sols nus, mais ne se voient pas sur des cartes de biomasse. La présence de denngo (Zornia glochidiata), extrêmement recherché par les éleveurs, influence l'itinéraire. Au Ferlo, les pasteurs augurent l'approche des pluies de la disparition de datjuki (la voie lactée) ainsi que de l'apparition des feuilles de eeri (Sclerocarya birrea).

\footnotetext{
${ }^{1}$ En puular.

${ }^{2}$ Ibid.
} 
L'unité pertinente des ressources pastorales est écologique et sociale : « un bon endroit » se distingue par l'ensemble des qualités de l'herbe, du sol, de l'air, par l'absence de parasites mais aussi par sa tranquillité : autrement dit, la gestion sociale des droits qui régulent son accès et sa jouissance.

Les éleveurs peuvent se renseigner sans se déplacer grâce aux téléphones portables et en observant l'état des animaux d'autrui au pied du forage, lieu de rassemblement en saison sèche. L'état des animaux inclut l'embonpoint, la vivacité, la brillance du poil ; connaître les marques des troupeaux et leurs propriétaires, les parcours habituels des voisins, permet d'en déduire l'abondance et la qualité des parcours utilisés. Leurs savoirs intègrent des connaissances sociales, zootechniques et écologiques. En comparaison, le moment d'intégration des disciplines au cours d'une recherche résulte à chaque fois d'un choix collectif compliqué.

\subsection{Une économie de ressources partagées}

Dans les sociétés pastorales, l'accès stratégique à l'eau et aux pâturages repose, quand tout va bien, sur la réciprocité des droits prioritaires. Les ayants droit locaux n'ont pas besoin de négocier l'accès à l'eau : l'accès des transhumants est régulé par la limite imposée à la durée de leur séjour. Ce mode de régulation ne doit pas être confondu avec l'accès libre aux points d'eau, qui n'est d'ailleurs pas originel mais résulte de l'impossibilité d'appliquer les règles d'appropriation, d'usage et d'entretien instaurées par les États indépendants, à l'époque de la création des points d'eau modernes (Thébaud, 2002). On se trouve à la fois en économie de partage où les parcours sont utilisés par plusieurs, dans un contexte où l'entraide face aux crises se restreint aux gens les plus proches (Sutter, 1987), et dans un espace où les infrastructures et l'arrivée de nouveaux groupes affaiblissent les anciennes obligations de réciprocité.

Dans la plupart des cas les populations pastorales ne sont pas ou plus « nomades » : au Niger, certains groupes Touaregs et Wodaabé sédentarisés de la région de Tahoua, dans l'Azawak, s'adonnent à l'agriculture depuis les années 1980 où ils ont perdu leur cheptel ; d'autres se déplacent pour la cure salée avec leurs troupeaux vers In-Gall, dans la région d'Agadez au Nord-Est du pays, et séjournent la plupart du temps à proximité de leur terroir d'attache, sauf en année difficile. Ces groupes gardent également en saison sèche des animaux appartenant à des villageois de la région, sur des pâturages loin des agglomérations.

La propension des populations pastorales à se rendre invisibles par la mobilité interstitielle est une stratégie connue depuis la période coloniale (Brémaud, 1955) : comportement pionnier ou fuite en avant, pour coloniser de nouvelles terres (Boutrais, 1973), échapper aux contraintes administratives, fiscales ou sanitaires (Kerven, 1992) ou aux habitudes prédatrices de représentants corrompus de l'administration (Diallo, 1994). Cette stratégie sociale, conjuguée à la gestion des ressources naturelles et aux systèmes de droits qui les régulent, ont créé des régimes de mobilité, une organisation familiale et sociale et des savoir-faire individuels qui sont aujourd'hui encore à la base de la reproduction des systèmes pastoraux. La gestion des ressources partagées s'est historiquement accommodée de politiques pastorales parfois ambitieuses mises en place sur la base de connaissances plus ou moins fines des réalités pastorales. Au Sénégal, des forages profonds jalonnent depuis les années 1950 le territoire du Ferlo tous les $30 \mathrm{~km}$, selon l'analyse des besoins journaliers et des capacités de déplacement des animaux. Ils ont modifié la mobilité sans conduire à la sédentarisation attendue des troupeaux. Et la réforme libérale de la gestion des forages étendue aux zones pastorales depuis les années 2000 a suscité chez les pasteurs des accommodements compatibles avec la transhumance (Ancey et al., 2008).

Cette stratégie d'évitement a sans doute permis aux pasteurs de préserver les bases économiques de leur mode de vie, nonobstant leur classement dans des catégories de pauvreté ou de vulnérabilité. Mais en conséquence, les populations pastorales, sédentarisées, transhumantes ou nomades, sont difficiles à localiser par l'administration et par les organismes qui interviennent en situation d'urgence.

\section{Des stratégies d'évitement des pasteurs à leur marginalisation}

\subsection{Une crise pastorale au Niger en 2005}

La marginalité des pasteurs transhumants sahéliens pendant les interventions d'aide à la fin des années 2000 renvoie d'abord aux systèmes d'information et d'alerte précoce ainsi qu'aux limites de coordination entre les opérateurs de l'aide. La distance structurelle entre le monde pastoral et les politiques de développement ne date pas des crises et traduit la difficulté pour des politiques économiques fondées sur des catégories comme l'État, la propriété, la sédentarité, à saisir les dynamiques pastorales. Le cas du Niger est exemplaire par la médiatisation, sinon l'acuité, de la crise alimentaire de 2005, par le rôle et les limites de son Dispositif national de prévention et de gestion des crises alimentaires (DNPGCA), le plus performant de la sous-région ; enfin, par l'ampleur des espaces arides et le poids démographique des populations pastorales sur son territoire.

Au Niger, la campagne agricole 2004-2005 s'annonçait difficile dès fin 2004, à cause d'une attaque acridienne et d'une mauvaise répartition des pluies pendant l'hivernage ; toutefois, les déficits céréaliers et fourragers n'étaient pas jugés exceptionnels. La crise a révélé les processus d'appauvrissement mettant en péril les structures et la reproduction sociale et économique des familles rurales et n'a fait qu'aggraver une situation structurellement critique dans le pays. La crise de 2005 fait écho aux précédentes. Témoin de la crise de 1985, l'anthropologue Spittler a traduit l'attitude morale, les perceptions et les stratégies des Touaregs Kel Ewey du Niger face aux sécheresses et aux famines: "Ce que signifie "exceptionnel" et "récurrent" ne peut être défini selon une chronologie absolue, mais d'après l'horizon d'expérience des personnes concernées » (Spittler, 1993). En août 2005, sous la pression d'une médiatisation internationale, le Programme alimentaire mondial (PAM), chef de file des organisations humanitaires dans le pays, obtenait l'accord du gouvernement pour déclencher des distributions alimentaires généralisées (Bouarfa et al., 2006). Mais au Nord de la région de Tahoua, sur les deux tiers du pays, les populations pastorales transhumantes dites «flottantes » par les opérateurs de l'aide et l'administration ne figuraient pas sur les recensements locaux dont disposaient les ONG mandatées pour distribuer l'aide alimentaire. Au moment où le DNPGCA a procédé au ciblage des zones et des populations vulnérables, puis élaboré le plan 
des distributions généralisées au Niger, de nombreuses familles pastorales résidentes depuis plusieurs années dans des terroirs villageois ciblés par l'aide, s'y acquittant de taxes locales, ont été administrativement invisibles aux yeux des distributeurs. Les transhumances et la vente sélective ont atténué les effets de la pénurie d'eau et de biomasse pour les plus grands éleveurs et pour ceux qui ont pu atteindre des pâturages arrosés, notamment vers l'ouest et le Mali. Les plus riches ont nourri et abreuvé leur troupeau au moyen de véhicules chargés d'aliments et de citernes d'eau. Une partie des familles pastorales a pu déployer des stratégies d'urgence : transhumances, migrations de travail en Algérie et en Libye ou dans les pays côtiers, de la Côte d'Ivoire au Gabon (Ancey, 2006). Les plus vulnérables ne disposant pas de bétail ou de maind'œuvre suffisants ont par contre subi les effets économiques, sanitaires et alimentaires de leur invisibilité pour les pouvoirs publics. L'analyse de la situation agroclimatique n'a pas pris en compte les inégalités d'accès aux ressources creusées par les décapitalisations en cheptel, en terres, en biens personnels, par les termes de l'échange. Ce n'est qu'en octobre 2005, après la fin des distributions généralisées, qu'une partie des populations pastorales a bénéficié d'un reliquat d'environ 400 tonnes de vivres. Tout s'est passé comme si les populations pastorales, généralement qualifiées de nomades, devenaient invisibles au niveau désagrégé du recensement, même lorsqu'elles résidaient dans des zones ciblées pour recevoir les distributions alimentaires. La qualification de la crise elle-même a été discutée. Alors que les organisations nationales et internationales présentes au Niger jugeaient la situation critique dès février-mars 2005, les références techniques (déficits céréaliers et fourragers) et les enjeux politiques (légitimité du pouvoir central liée à sa fonction nourricière ; Arditi et al., 2011) ont retardé la qualification de «famine » par le Premier ministre jusqu'en août 2005 ; ce terme n'a d'ailleurs été que temporairement employé avant d'être remplacé définitivement par celui de crise alimentaire.

\subsection{Des systèmes d'information en évolution, mais qui ne parviennent pas à rendre compte des réalités pastorales}

Suite aux crises des années 1973-1974 et 1984-1985, et à l'évolution des conceptions de la sécurité alimentaire mettant alors l'accent sur l'accessibilité de la nourriture, des systèmes d'information sur les marchés (SIM) ont été conçus au Sahel par les administrations nationales et les bailleurs de fonds internationaux pour prévenir et gérer les crises alimentaires en améliorant le fonctionnement des marchés. On définit ici un système d'information comme un ensemble organisé de ressources (matérielles et humaines) qui permet de classifier, traiter et diffuser de l'information dans un environnement donné. Le développement des techniques d'alerte précoce visait à dépasser les limites de la statistique agricole par des indicateurs climatiques et qualitatifs. Conçus comme des outils d'appui aux politiques de filières, les systèmes de suivi et d'alerte conjugués ont très vite servi essentiellement à construire des bilans céréaliers régionaux utiles aux agences d'aide et, en période de déficit, à faciliter la délivrance de l'aide alimentaire (Egg et Gabas, 1997). L'inclusion de l'aide au cœur de la définition de la sécurité alimentaire (Maxwell et
Frankenberger, 1992) converge avec l'orientation donnée à ces outils et leur perfectionnement. Ils représentent un monde rural fondamentalement agricole et sédentaire, caractérisé par la faiblesse de ses ressources et sa pauvreté.

La plupart des systèmes d'alerte précoce (SAP) se basent sur des données pluviométriques et des modèles de simulation de production de biomasse. Or, la différence entre la production réelle et les chiffres estimés est évaluée à plus ou moins $25 \%$ (Van Dijk, 1997). De plus, dans les zones pastorales arrosées de moins de $400 \mathrm{~mm}$ de pluies annuelles, le rôle de la production céréalière dans l'économie familiale et dans l'alimentation est beaucoup plus réduit qu'en zone agricole. Les systèmes d'information sur la sécurité alimentaire (SISA), et les SAP existants depuis les années 1980 avaient une couverture satellitaire ciblée sur les zones de production agricole, leurs principaux indicateurs étaient conçus pour mesurer des besoins céréaliers; ils n'étaient donc pas conçus pour évaluer les conditions de vie des populations en zones arides, en tenant compte de la complexité des contraintes et des stratégies à l'échelle régionale ou familiale. Enfin, comme la fiabilité du pronostic décroît avec l'échelle spatiale, les SAP et les systèmes d'information couvrant les productions ou les marchés étaient peu fonctionnels à l'échelle locale qui est celle des organisations de producteurs et de la gestion des territoires. Certains d'entre eux ont été perfectionnés au cours des années 2000 par l'introduction d'indicateurs d'aptitudes, de termes de l'échange entre céréales et petit bétail, et de mouvements de population pour rendre mieux compte des conditions de vie et du niveau de vulnérabilité. Cependant, en 2004 et 2005, des indications qualitatives figurant dans les bulletins régionaux du «Famine Early Warning System » (FEWS) publiés par le centre Agrhymet n'ont pas été prises en compte dans le diagnostic de la situation.

En résumé, les dispositifs d'information ont été perfectionnés (Franck Galtier et al., 2014) pour contribuer à la prévention des crises alimentaires, en lien avec le renouvellement des conceptions de la sécurité alimentaire et l'émergence des initiatives humanitaires. Cependant, la fonction initiale de ces outils, leur usage, leurs caractéristiques techniques n'ont guère permis de mettre en évidence la situation pastorale à des moments critiques.

\subsection{Des systèmes d'information mis au service de la capacité autorégulatrice des marchés}

L'expansion des différents systèmes d'information (SIM, SI, SAP) en Afrique sahélienne, impulsée par l'impact international des crises alimentaires et des sécheresses des années 1970 et 1980, a bénéficié du développement de la statistique et de l'informatique, qui a permis de perfectionner le suivi des groupes à risques partout dans le monde (Castel, 1981). Au Sahel, cette évolution est marquée par des dispositifs successifs mis en place après les programmes d'ajustement structurel : enquêtes lourdes à plusieurs passages pour mesurer les conséquences sociales de l'ajustement, puis enquêtes légères et rapides plus ou moins participatives, enfin, usage croissant des images satellitaires supposées moins coûteuses et plus précises que les enquêtes de terrain. Dans les représentations prévalant dans les organisations internationales, les sécheresses avaient conforté une image des régions subsahariennes structurellement exposées 
aux pénuries alimentaires, dans un contexte de marchés agricoles caractérisés par les excédents céréaliers des pays industrialisés. Les systèmes d'information mettaient donc logiquement l'accent sur la disponibilité des récoltes ou des biens céréaliers et leur accessibilité économique dans les neuf États membres du Comité permanent inter-États de lutte contre la sécheresse au Sahel (CILSS).

Dans les années 1980 et 1990, la géopolitique internationale a exacerbé la croyance en la capacité autorégulatrice du marché et son rôle dans la fourniture de biens publics, $y$ compris la sécurité alimentaire, et stimulé la recherche sur ces enjeux. De nombreuses interventions pour la sécurité alimentaire et la prospérité économique ont tenté d'améliorer les performances, la transparence et la concurrence sur les marchés, en fluidifiant les échanges et en diffusant une bonne information sur les prix. Observatoires et études ont été lancés pour remplacer les offices nationaux de gestion des marchés céréaliers au Sahel, supprimés par les Programmes d'ajustement structurel.

Suivant le dogme économiciste qui centre l'économie sur les échanges, et étend ses analyses aux comportements de la vie sociale, si les populations pastorales étaient mal connues des économistes et des politiques, c'est parce qu'elles vivaient en quasi-autarcie, hors marché. "Nomads are a nuisance, and not only to a centralising State: they are awkward even for theory... »(Gellner, 1981, cité par Pouillon [1990]). Le rôle crucial des échanges entre les économies pastorales et « l'extérieur » est désormais reconnu (Khazanov, 1984; Kerven, 1992 ; Wane et al., 2010), qu'il s'agisse de razzias, d'échanges sur les marchés, d'interactions avec les agriculteurs ou encore de migrations. Les capacités d'adaptation pastorales sont démontrées non seulement face aux aléas naturels, mais aussi face aux opportunités économiques et techniques.

La marginalité pastorale tient non seulement aux limites des outils d'informations mais aussi à des habitudes cognitives et politiques chez tous les acteurs. Il convient de ne pas omettre la spécificité de la situation sahélienne liée à la faiblesse structurelle des administrations nationales et à un marché du développement aux acteurs pléthoriques : institutions et centres de recherche, organisations non gouvernementales, coopération décentralisée... La production, la transmission et le traitement des données deviennent des enjeux de transaction. En pratique, les agents de base manquent de moyens de déplacement pour enquêter; les données transmises aux niveaux intermédiaires sont donc souvent fabriquées au jugé, avant de faire l'objet d'une synthèse autant technique que politique. Dans ce contexte, des observateurs font l'hypothèse que les réformes de gouvernance ne sont guère à attendre « par en haut », mais plutôt « par le milieu », au niveau de l'appui aux cadres, et « par le bas », au niveau des services élémentaires de l'État ou sous la pression des usagers (Olivier de Sardan, 2004).

\section{Conclusion}

Les logiques économiques et écologiques pastorales de plus de 20 millions de pasteurs restent méconnues des politiques alors que le Sahel a été précurseur sur le continent dans le domaine de l'information sur la sécurité alimentaire (Egg et Gabas, 1997). Le poids démographique des populations pastorales et de leurs troupeaux s'est accru grâce à leurs capacités adaptatives et à des décennies d'interventions dans les domaines hydrauliques et vétérinaires, leur apport économique pour le continent est démontré (Hatfield et al., 2006). Aujourd'hui la marginalité des pasteurs aggrave non seulement la situation des plus vulnérables, mais dessert les éleveurs moins vulnérables et compromet les dynamiques rurales et l'approvisionnement urbain. Les mécanismes collectifs de sécurisation tels que la mobilité et la réciprocité de l'accès aux ressources nécessitent d'être compris et accompagnés par les politiques. Cela suppose la révision de certains principes agronomiques d'intensification fondés sur une activité sédentaire dans un milieu stabilisé, voire artificialisé ; et la remise en cause de l'idéologie liant la sécurité foncière à l'appropriation exclusive des ressources naturelles (terre, eau, biomasse) formalisée par des titres.

Pour autant, ces changements cognitifs nécessaires ne suffiraient pas sans que soient surmontés d'autres hiatus politiques. Le contexte actuel exacerbe la redistribution des rôles du public vers le privé (associations, ONG, fondations) amorcée par la libéralisation des politiques dans les années 1980 ; l'enjeu sécuritaire stimule de nouvelles actions au Sahel (en 2013, conférence internationale au Tchad «Pastoralisme et sécurité au Sahel » financée par l'Agence française de développement, Forum international sur le pastoralisme en Mauritanie financé par le CILSS et la Banque mondiale, création du G5 [Tchad, Mali, Niger, Mauritanie et Burkina Faso]). La coordination entre opérateurs devient un enjeu majeur, sans parler de leur " alignement » en cohérence avec des stratégies politiques, requis depuis une dizaine d'années (Déclaration de Paris, 2005). Ces initiatives, pour la plupart régionales, auront plus de portée si les politiques nationales sur lesquelles elles s'appuient prennent davantage en compte les enjeux de l'élevage mobile et la mise en valeur pastorale des ressources.

La marginalité des populations pastorales et leur propension à se rendre invisibles restent souvent analysées avec ambivalence : on critique leur marginalité dans les actions de développement, tout en concédant que leur invisibilité les a préservées des effets nocifs d'interventions politiques contradictoires avec leurs processus de reproduction sociale. Cependant, les capacités d'adaptation des populations pastorales en Afrique subsaharienne, longtemps liées à leur stratégie d'évitement des pressions, sont aujourd'hui en contradiction avec une action collective susceptible de peser sur leur devenir citoyen au sein des sociétés auxquelles elles appartiennent.

Remerciements. Je remercie les trois lecteurs anonymes pour leurs commentaires, qui ont permis d'améliorer une première version de ce texte.

\section{Références}

Ancey V. 2006. Évaluation de la réponse du PAM à la crise alimentaire au Niger en 2005. Note 4 : caractérisation de la crise pastorale. Rapport technique PAM, Rome.

Ancey V, Fréguin-Gresh S. 2014. Les familles, le travail et l'exploitation agricole. In : Sourisseau J-M, ed. Agricultures familiales et mondes à venir. Versailles : Quae, pp. 61-75.

Ancey V, Wane A, Muller A, André D, Leclerc G. 2008. Payer l'eau au Ferlo : stratégies pastorales de gestion communautaire de l'eau. Autrepart 46: 51-66. 
Andrieu N, Josien E, Duru M. 2007. Relationships between diversity of grassland vegetation, field characteristics and land use management practices assessed at the farm level. Agric Ecosyst Environ 120: 359-369.

Arditi C, Janin P, Marie A. 2011. La lutte contre l'insécurité alimentaire au Mali : réalités et faux-semblants, Paris : Karthala.

Behnke R, Scoones I. 1992. Rethinking rangeland ecology. Drylands Issue Pap 33: 33.

Bouarfa Y, Blein R, Girerd-Barclay E, Ancey V, Mossi I. 2006. Évaluation de la réponse du PAM à la crise alimentaire au Niger en 2005, Roma : World Food Program.

Boutrais J. 1973. La colonisation des plaines par les montagnards au nord du Cameroun (Monts Mandara), Bondy : IRD Editions.

BrémaudO. 1955. Nomadisme et transhumance en Afrique subsaharienne. Les mouvements pastoraux dans les parcours extrêmes-orientaux du Soudan français. Rev El Méd Vét Pays Trop 8(4): 327-355.

Castel R. 1981. La gestion des risques : de l'anti-psychiatrie à l'aprèspsychanalyse, Paris : Éditions de Minuit.

Couty P. 1984. La vérité doit être construite. Cah ORSTOM Sér Sci Hum XX(1): 5-15.

Craine JM, Ocheltree TW, Nippert JB, et al. 2013. Global diversity of drought tolerance and grassland climate-change resilience. Nat Clim Chang 3: 63-67.

Desrosières A. 2010. La politique des grands nombres : histoire de la raison statistique, Paris : La Découverte.

Diallo Y. 1994. Barani : une chefferie satellite des grands États du $\mathrm{XIX}^{\mathrm{e}}$ siècle (Barani, a chiefdom in the orbit of major 19th-century state formations). Cah Études Afr 34: 359-384.

Egg J, Gabas J-J. 1997. La prévention des crises alimentaires au Sahel et le rôle des dispositifs d'information. Stateco 87-88: 9-27.

Egg J, Michiels D, Blein R, Vanessa AF. 2006. Évaluation du dispositif de prévention et de gestion des crises alimentaires du Niger : rapport principal, Paris : IRAM.

Figuié M, Hubert B. 2012. Pour qui, pourquoi une ressource est-elle jugée dégradée ? Pâturages et agriculture familiale au Brésil. Nat Sci Soc 20: 297-309.

Franck Galtier David-Benz H, Subervie J, Egg J. 2014. Les systèmes d'information sur les marchés agricoles dans les pays en développement : nouveaux modèles, nouveaux impacts. Cah Agric 23(4-5): 245-258.

Gastellu J-M, Dubois J-L. 1997. En économie : l'unité retrouvée, la théorie revisitée. Approches des dynamiques contemporaines. In : Pilon M, Locoh T, Vignikin E, Patrice V, eds. Ménages et familles en Afrique. Paris : CEPED, pp. 75-98.

Gellner E. 1981. Anomalies of no fixed abode. Review of nomads of South Siberia: the pastoral economics of Tuva, by Sevyan Vainshtein. Times Lit Suppl 273.

Hatfield R, Davies J, Wane A, et al. 2006. Global review of the economics of pastoralism. In: World Initiative for Sustainable Pastoralism/IUCN, Nairobi, Kenya.

Hubert B. 2012. Préface. In : Toutain B, Marty A, Bourgeot A, Ickowicz A, Lhoste P, eds. Pastoralisme en zone sèche. Le cas de l'Afrique subsaharienne. Montpellier : CFSD/Agropolis International, pp. 2-3.

Janin P, Martin-Prevel Y. 2006. Des indicateurs à l'action : vulnérabilité alimentaire et situation nutritionnelle en milieu rural sahélien burkinabé. Can J Afr Stud / Rev Can Études Afr 40: 443-461.

Kerven C. 1992. Customary commerce. A historical reassessment of pastoral livestock marketing, London: Overseas Development Institute.
Khazanov AM. 1984. Nomads and the outside world, Cambridge: Cambridge University Press.

Krätli S. 2015. Valuing variability: new perspectives on climate resilient drylands development, London: IIED.

Krätli S, Schareika N. 2010. Living off uncertainty: the intelligent animal production of dryland pastoralists. Eur $J$ Dev Res 22: 605-622.

Marty A. 1993. La gestion des terroirs et les éleveurs : un outil d'exclusion ou de négociation ? Rev Tiers Monde 34(134): 327-344

Maxwell S, Frankenberger T. 1992. Household food security: concepts, indicators and measurements: a technical review. New York and Rome: United Nations Children's Fund (UNICEF) and International Fund for Agricultural Development (IFAD).

Meillassoux C. 1975. Femmes, greniers et capitaux, Paris : F. Maspero.

Olivier de Sardan J-P. 2004. État, bureaucratie et gouvernance en Afrique de l'Ouest francophone. Polit Afr 4(96): 139-162.

Olivier de Sardan J-P. 2007. Analyse rétrospective de la crise alimentaire au Niger en 2005, Niger : LASDEL.

Olivier de Sardan J-P. 2008. La crise alimentaire au Niger. Afr Contemp 1(225): 17-294.

Pouillon F. 1990. Sociétés pastorales et développement : histoire des politiques et critique des doctrines : présentation. Cah Sci Hum 26: 3-7.

Scoones I. 1999. Nouvelles orientations du développement pastoral en Afrique : vivre dans un environnement incertain, Paris : Karthala.

Spittler G. 1993. Les Touaregs face aux sécheresses et aux famines : les Kel Ewey de l'Aïr (Niger) ; (1900-1985). Paris : Karthala.

Steinfeld H, Mooney HA, Schneider F, Neville LE. 2013. Livestock in a changing landscape, volume 1: drivers, consequences, and responses, Washington, D.C.: Island Press.

Sullivan S, Rohde R. 2002. On non-equilibrium in arid and semi-arid grazing systems. J Biogeogr 29: 1595-1618.

Sutter J. 1987. Cattle and inequality: herd size differences and pastoral production among the Fulani of northern Senegal. Africa 57: 196-218.

Swift J. 2001. Pastoralism and mobility in the drylands. Available by challenge paper, the global drylands imperative. Available from http://www.undp.org/drylands/policy-cpapers.htm.

Thébaud B, ed. 2002. Foncier pastoral et gestion de l'espace au Sahel : Peuls du Niger oriental et du Yagha burkinabé, Paris : Karthala.

Thébaud B, Grell H, Miehe S. 1995. Vers une reconnaissance de l'efficacité pastorale traditionnelle : les leçons d'une expérience de pâturage contrôlé dans le nord du Sénégal, London : International Institute for Environment and Development (IIED).

Thornton P, Van de Steeg J, Notenbaert A, Herrero M. 2009. The impacts of climate change on livestock and livestock systems in developing countries: a review of what we know and what we need to know. Agric Syst 101: 113-127.

Van Dijk H. 1997. Risk, agro-pastoral decision-making and natural resource management in Fulbe Society, Central Mali. Nomadic Peoples 1: 108-132.

Verschuur C, Guérin I, Guétat-Bernard H. 2015. Sous le développement, le genre, Marseille : IRD.

Wane A, Ancey V, Touré I. 2010. Pastoralisme et recours aux marchés : cas du Sahel sénégalais (Ferlo) (Sahelian herders and the markets: evidence from the Senegalese Sahel (Ferlo)). Cah Agric 19: 14-20.

Citation de l'article : Ancey V. 2016. Comment se construit l'invisibilité des populations pastorales au Sahel : l'expérience de la crise alimentaire de 2005 au Niger. Cah. Agric. 25: 55003. 